\title{
Transfert du ${ }^{65} \mathrm{Zn}$ de l'eau aux parties aériennes du maïs et du haricot irrigués par aspersion $\left(^{*}\right)$
}

\author{
INFLUENCE DE LA FORME CHIMIQUE DU POLLUANT \\ ET DE LA QUALITÉ DE L'EAU
}

J. DELMAS, R. DISDIER, A. GRAUBY

(Manuscrit reçu le 21 novembre 1977)

\begin{abstract}
RÉSUMẾ
L'influence de la qualité de l'eau d'irrigation et de la forme chimique du ${ }^{65} \mathrm{Zn}$, présent dans cette eau, sur son transfert aux plantes cultivées (haricot, mals) est étudiée aux cours d'expérimentations en conditions contrôlées qui simulent l'irrigation par aspersion. Deux formes chimiques du polluant $\left(\mathrm{Cl}_{2} \mathrm{Zn}\right.$ et $\mathrm{Zn}$ EDTA) sont additionnées à uné eau déminéralisée et une eau de rivière. L'influence de la présence dans l'eau d'irrigation du $\mathrm{Cd}$ et du $\mathrm{Zn}$ stables sur le transfert direct du ${ }^{65} \mathrm{Zn}$ au mais est également étudiée. Les facteurs de transfert du ${ }^{69} \mathrm{Zn}$ et du cadmlum sont évalués. L'incidence des résultats obtenus quant à la protection du consommateur final est discutée.
\end{abstract}

\begin{abstract}
The effect of the quality of irrigation water and of the chemical form of ${ }^{65} \mathrm{Zn}$ available in the water on its transfer to crops (beans, corn) was studied during experiments under controlled conditions simulating spray irrigation. Two chemical forms $\left(\mathrm{Cl}_{2} \mathrm{Zn}\right.$ and $\mathrm{Zn}$ EDTA $)$ were added to demineralized and river water. The effect of stable $\mathrm{Cd}$ and $\mathrm{Zn}$ present in irrigation water on the direct transfer of ${ }^{65} \mathrm{Zn}$ to corn was also studied. Transfer factors for ${ }^{65} \mathrm{Zn}$ and cadmium were evaluated. The consequences as to the protection of the final consumer are discussed.
\end{abstract}

(*) Travail effectué dans le cadre de l'association Euratom/C.E.A., Contrat VEN n 0693.

(**) Commissariat à l'Énergie atomique, Institut de Protection et de Sûreté nucléaire, Département de Protection, Service d'Études et de Recherches sur l'Environnement, Section de Radioécologie, B.P. 1, 13115 Saint-Paul-Les-Durance.

RADIOPROTECTION, VOL. $13-\mathrm{N}^{\circ} 1$ 


\section{INTRODUCTION}

Le ${ }^{65} \mathrm{Zn}$ est un des radionucléides pouvant se trouver dans les effluents liquides rejetés en rivière par les installations nucléaires. En radioécologie, il paraît important pour différentes raisons : par ses caractéristiques nucléaires, par le rôle du zinc dans le milieu vivant, et par la complexité de ses propriétés chimiques.

Son transfert de l'eau d'irrigation aux produits agricoles a été mis en évidence par des chercheurs de Hanford [1-2]. Ils ont montré que les facteurs de transfert $a$ pouvaient varier de façon considérable avec le type de végétal et le mode d'apport. Le transfert est faible pour la vigne et le blé $(a=$ respectivement 0,47 et 0,83 ). Il est faible également pour la tomate : $a=2,4$, et élevé pour l'herbe de pâturage : $a=440$. Dans ce dernier cas, l'absorption foliaire est vraisemblablement à l'origine de ce transfert relativement important. Nous avons donc voulu préciser certains aspects du mode de transfert du ${ }^{65} \mathrm{Zn}$ à la suite d'irrigation contaminante.

La mobilité du ${ }^{65} \mathrm{Zn}$ dans les divers écosystèmes concernés par les rejets dépend notamment de ses formes chimiques dans.l'eau de rivière. Ces formes chimiques résultent des traitements appliqués en usine et de la qualité de l'eau. Pour ces raisons, dans le cadre des travaux sur la contamination radioactive de la chaîne alimentaire par les eaux d'arrosages éventuellement polluées, nous avons étudié le transfert du ${ }^{65} \mathrm{Zn}$ sous forme ionique ou complexée en fonction de la qualité de l'eau.

Il est bien connu également que des éléments homologues peuvent influencer le transfert des éléments traces dans le milieu. Aussi nous avons, pour compléter ces travaux, étudié également l'influence du cadmium et du zinc stables, éléments fréquemment rejetés en rivière, sur le transfert du ${ }^{65} \mathrm{Zn}$.

\section{ORGANISATION DE L'EXPÉRIENCE}

L'objectif de l'expérimentation est de préciser les facteurs de transfert du ${ }^{65} \mathrm{Zn}$ de l'eau d'arrosage aux parties aériennes des plantes irriguées par aspersion sous l'influence de ces divers paramètres :

- formes chimiques du radionucléide;

- qualité de l'eau;

- présence dans l'eau des éléments stables du cadmium et du zinc.

Par ailleurs, afin d'obtenir des facteurs de transfert proches de ceux du milieu exploité par l'homme, le modèle expérimental a été conçu de façon à simuler au mieux les conditions de la pratique. Dans ce but, l'irrigation par aspersion entraînant la contamination directe du maīs a été pratiquée en chambre climatique de grandes dimensions [3]. La culture des plantes a été menée dans des bacs de $1 \mathrm{~m}^{2}$ de section remplis de terre sur une hauteur de $0,50 \mathrm{~m}$. Cette pratique permet d'avoir des conditions de croissance reproductibles et un développement équilibré des végétaux. Par ailleurs, il est possible de mettre en cuvre des quantités d'eau équivalentes à celles des irrigations de plein champ. Il en est de même pour la distribution de l'eau pendant la croissance des plantes. 
Le maĩs et le haricot ont été choisis en se basant sur les critères suivants :

- importance dans l'agriculture;

- large utilisation dans l'alimentation du bétail ou de l'homme;

- besoin en eau en cours de culture.

Par bac ont été mis en place 6 plants de maĩs de la variété « Iowa 44-17 \# ou 8 plants de haricots de la variété « Contender $"$; tous ces plants ont été conduits à maturité complète, stade auquel les récoltes ont été effectuées.

Deux types d'eau ont été utilisés :

- de l'eau déminéralisée à $\mathrm{pH} 5$;

- de l'eau du Rhône à pH 8,2 avec une teneur en $\mathrm{Ca}^{++}$relativement élevée (tableau I).

Dans une série d'essais le ${ }^{65} \mathrm{Zn}$ ajouté à l'eau était sous 2 formes : $\mathrm{Cl}_{2} \mathrm{Zn}$ et complexé à l'EDTA. Dans cet essai; les pulvérisations ont été appliquées sur des mails et des haricots.

\section{TABLEAU I}

Caractéristiques du sol, de l'eau de rivière, du climat

\begin{tabular}{|c|c|c|c|}
\hline $\begin{array}{c}\text { Sol } \\
\text { Caractéristiques } \\
\text { physicochimiques }\end{array}$ & & $\begin{array}{l}\text { Eau d'aspersion } \\
\text { Caractéristiques }\end{array}$ & \\
\hline Sable grossier $(\%) \ldots \ldots \ldots \ldots \ldots$ & 14 & 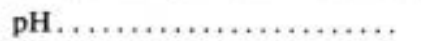 & 8,2 \\
\hline Sable fin $(\%) \ldots \ldots \ldots \ldots \ldots \ldots$ & 14 & Résidu $\sec (g / l) \ldots \ldots \ldots \ldots \ldots$ & 0,34 \\
\hline Limon grossier $(\%) \ldots \ldots \ldots \ldots \ldots$ & 12 & Résistivité $(\Omega / \mathrm{cm}) \ldots \ldots \ldots \ldots$ & 2770 \\
\hline Argile $(\% \ldots \ldots \ldots \ldots \ldots . . . . . . .$. & 9,4 & $\mathrm{Ca}(\mathrm{mg} / \mathrm{l}) \ldots \ldots \ldots \ldots \ldots \ldots$ & 65 \\
\hline Calcaire total $(\% \ldots \ldots \ldots \ldots$ & 40 & $\mathrm{Mg}(\mathrm{mg} / \mathrm{l}) \ldots \ldots \ldots \ldots \ldots$ & 6,7 \\
\hline $\begin{array}{l}\text { Calcaire actif }(\%, \ldots \ldots \ldots \ldots \ldots \\
\text { (méthode à l'oxalate) }\end{array}$ & 40 & $\begin{array}{l}\mathrm{Na}(\mathrm{mg} / \mathrm{l}) \ldots \ldots \ldots \ldots \ldots \ldots \ldots \ldots \ldots \ldots \\
\mathrm{K}(\mathrm{mg} / \mathrm{l}) \ldots \ldots \ldots \ldots \ldots \ldots \ldots\end{array}$ & $\begin{array}{r}14,6 \\
2,2\end{array}$ \\
\hline pH cau $\ldots \ldots \ldots \ldots \ldots \ldots \ldots$, & 8,7 & $\mathrm{Zn}(\mu \mathrm{g} / \mathrm{I}) \ldots \ldots \ldots \ldots \ldots \ldots$ & 40 \\
\hline Matières organiques $(\%) \ldots \ldots \ldots$. & 1 & Bicarbonates $(\mathrm{mg} / \mathrm{l}) \ldots \ldots \ldots \ldots$ & 140 \\
\hline Capacité d'échange & & Chlorures $(\mathrm{mg} / \mathrm{l}) \ldots \ldots \ldots \ldots$ & 32,3 \\
\hline$(m e q / 100 \mathrm{~g}) \ldots \ldots \ldots \ldots \ldots \ldots$ & 33,4 & Sulfates $(\mathrm{mg} / \mathrm{l}) \ldots \ldots \ldots \ldots \ldots$ & 69 \\
\hline $\begin{array}{l}\text { Bases échangeables } \\
\text { a pH } 7 \text { (acétate } \mathrm{NH}_{4} \text { ) : }\end{array}$ & & $\begin{array}{l}\text { Nitrates }(\mathrm{mg} / \mathrm{l}) \ldots \ldots \ldots \ldots \ldots \\
\mathrm{Cd}(\mu \mathrm{g} / \mathrm{l}) \ldots \ldots \ldots \ldots \ldots \ldots\end{array}$ & $\begin{aligned} & 3,4 \\
< & 4\end{aligned}$ \\
\hline $\mathrm{Ca}(\mathrm{meq} / 100 \mathrm{~g}) \ldots \ldots \ldots \ldots \ldots \ldots$ & 32,7 & Densité d'arrosage $(\mathrm{mm} / \mathrm{h}) \ldots$ & 9,9 \\
\hline $\mathrm{Mg}(\mathrm{meq} / 100 \mathrm{~g}) \ldots \ldots \ldots \ldots \ldots$ & 0,6 & & à 10,3 \\
\hline $\mathrm{K}(\mathrm{meq} / 100 \mathrm{~g}) \ldots \ldots \ldots \ldots \ldots \ldots$ & 0,09 & Dose $(\mathrm{mm}) \ldots \ldots \ldots \ldots \ldots \ldots$ & 50 \\
\hline $\mathrm{Na}(\mathrm{meq} / 100 \mathrm{~g}), \ldots \ldots \ldots \ldots \ldots$ & 0,05 & Intervalle (jours)........... & 8 \\
\hline
\end{tabular}

Dans une autre série d'essais, des arrosages ont mis en cuvre de l'eau de rivière additionnée d'une part de $5 \mathrm{mg} / \mathrm{l}$ de zinc stable, et d'autre part de cadmium stable aux concentrations de 10 et $50 \mathrm{ppb}$ soit, respectivement, 0,010 et $0,050 \mathrm{mg} / \mathrm{l}$. Ces concentrations ont été choisies en se référant aux concentrations limites admises [5]. La concentration maximale admissible du

voL. $13-\mathrm{N}^{\circ} 1$ 
cadmium pour l'eau de boisson est $0,010 \mathrm{mg} / \mathrm{l}$. Pour le zinc, les normes OMS pour l'eau de boisson en 1965 sont :

- concentration maximale acceptable : $5 \mathrm{mg} / \mathrm{l}$;

- concentration maximale admissible : $15 \mathrm{mg} / \mathrm{l}$.

Les limites de concentration recommandées pour les eaux d'irrigation sont les suivantes [6] :

- en irrigation continue et intense : $5 \cdot 10^{-9}\left(5 \cdot 10^{-3} \mathrm{mg} / \mathrm{l}\right)$ pour le cadmium et $5 \cdot 10^{-6}(5 \mathrm{mg} / \mathrm{l})$ pour le zinc;

- en irrigation discontinue : $50.10^{-9}\left(50.10^{-3} \mathrm{mg} / \mathrm{l}\right)$ pour le cadmium et $10.10^{-6}(10 \mathrm{mg} / \mathrm{l})$ pour le zinc, avec possibilité, pour les irrigations peu intenses sur sols relativement lourds, de dépasser ces valeurs.

Dans la première série d'essais où étaient mises en cuvre de l'eau déminéralisée et de l'eau de rivière, il n'y avait pas de protection du sol. Dans la deuxième série d'essais pour éviter l'incidence de la contamination indirecte par voie racinaire, le sol était protégé par une feuille de polyvinyle dont les interstices à la base des plants étaient soigneusement rendus étanches. L'emploi de ces deux procédés devait nous permettre d'évaluer l'importance respective des contaminations directe et indirecte.

Le tableau I présente l'ensemble des conditions expérimentales.

La préparation des échantillons et les mesures radioactives ont été effectuées suivant des méthodes classiques. Les feuilles et les grains ont été séchés et broyés. La poudre ainsi obtenue minéralisée à $60-70^{\circ} \mathrm{C}$ avec $\mathrm{HNO}_{3} 6 \mathrm{~N}$ a été analysée par absorption atomique pour la recherche du cadmium.

Le ${ }^{65} \mathrm{Zn}$ a été déterminé par spectrométrie $\gamma$ à l'aide d'un sélecteur Intertechnique.

Au cours des essais présentés dans ce rapport, nous avons constaté que les teneurs en zinc stable des feuilles et des grains du maïs témoins sont normales. Les végétaux ne sont pas carencés (tableau II).

TABLEAU II

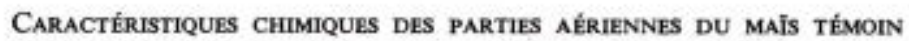

\begin{tabular}{|c|c|c|c|c|c|c|}
\hline & $\underset{(\mathrm{mg} / \mathrm{g})}{\mathrm{Ca}}$ & $\underset{(\mathrm{mg} / \mathrm{g})}{\mathrm{Cd}}$ & $\underset{(\mathrm{mg} / \mathrm{g})}{\mathbf{K}}$ & $\underset{(\mathrm{mg} / \mathrm{g})}{\mathrm{Mg}}$ & $\underset{(\mathrm{mg} / \mathrm{g})}{\mathrm{Na}}$ & $\underset{(\mathrm{mg} / \mathrm{g})}{\mathrm{Zn}}$ \\
\hline Feuille & 19 & $<0,0005$ & 1,20 & 1,48 & 0,507 & 0,066 \\
\hline Grains & 5,5 & $<0,0005$ & 4,30 & 1,19 & 0,124 & 0,027 \\
\hline
\end{tabular}

\section{TRANSFERT DU ${ }^{65} \mathrm{Zn}$}

Les tableaux III et IV présentent les facteurs de transfert du ${ }^{65} \mathrm{Zn}$ de l'eau au végétal pour les différents organes à la récolte

$$
a=\frac{\mathrm{pCi} / \mathrm{kg} \text { frais }}{\mathrm{pCi} / \mathrm{l}} \text {. }
$$


Ce rapport indique que $1 \mathrm{~kg}$ de produit frais contient une quantité de radionucléide équivalente à celle contenue dans $($ a $a)$ litres d'eau d'aspersion.

TABLEAU III

Facteurs de transfert dU ${ }^{65} \mathrm{Zn}$ aU Maĩs et aU haricot

\begin{tabular}{|c|c|c|c|c|c|}
\hline \multirow[t]{3}{*}{. } & & \multicolumn{4}{|c|}{$\begin{array}{l}\text { Taux de transfert } a \\
\frac{\mathrm{pCi} / \mathrm{kg} \text { frais }}{\mathrm{pCi} / \mathrm{l} \text { eau }}\end{array}$} \\
\hline & & \multicolumn{2}{|c|}{$\begin{array}{c}\text { Eau } \\
\text { déminéralisée }\end{array}$} & \multicolumn{2}{|c|}{$\begin{array}{c}\text { Eau } \\
\text { de rivière }\end{array}$} \\
\hline & & ${ }^{65} \mathrm{ZnCl}_{2}$ & $\begin{array}{c}{ }^{65} \mathrm{Zn} \\
\text { EDTA }\end{array}$ & ${ }^{65} \mathrm{ZnCl}_{2}$ & $\begin{array}{l}{ }^{65} \mathrm{Zn} \\
\text { EDTA }\end{array}$ \\
\hline \multirow{2}{*}{$\begin{array}{c}\text { Haricots } \\
\text { Volume eau distribuée : } \\
150 \mathrm{l} / \mathrm{m}^{2}\end{array}$} & Feuilles & 7,3 & 4,8 & 10,1 & 8,5 \\
\hline & Gousses & 0,5 & 0,5 & 0,6 & 0,5 \\
\hline \multirow[t]{2}{*}{$\begin{array}{c}\text { Mals } \\
\text { Volume eau distribuée : } \\
400 \mathrm{l} / \mathrm{m}^{2}\end{array}$} & $\begin{array}{l}\text { Feuilles } \\
\text { sans } \\
\text { gaines }\end{array}$ & 8,6 & 15,2 & 20,1 & 26,6 \\
\hline & Grains & 3,2 & 4,2 & 4,2 & 4,7 \\
\hline
\end{tabular}

Ce tableau permet de mettre en évidence l'influence sur le transfert du ${ }^{65} \mathrm{Zn}$ de différents paramètres :

- partie et nature du végétal;

- formes chimiques du radionucléide et qualité de l'eau.

Les facteurs de transfert observés ici, sur les feuilles et les fruits, sont différents. Les facteurs de transfert du ${ }^{65} \mathrm{Zn}$ sont plus élevés pour les feuilles des deux espèces que pour les grains de maĩs et les gousses de haricot. Les gousses, qui n'apparaissent que tardivement en cours de croissance, sont moins soumises à la contamination par contact direct avec l'eau que les feuilles. Par contre, les grains de maĩs protégés par les spathes sont plus contaminés que les gousses, proportionnellement à la contamination des feuilles respectives. On peut donc penser à une certaine mobilité du ${ }^{65} \mathrm{Zn}$ qui serait plus importante dans le maĩs que dans le haricot. Les facteurs de transfert du ${ }^{65} \mathrm{Zn}$ observés dans ces essais sont plus élevés que ceux que nous avons observés sur la salade [3], mais ceux-ci avaient été relevés sur des végétaux lavés avant les mesures spectrométriques. Le lavage a, sans doute, éliminé partiellement le ${ }^{65} \mathrm{Zn}$.

Cependant, des pommes soumises à des conditions de contamination et des traitements d'échantillons identiques à celles mises en œuvre dans cet essai, donnaient lieu à des facteurs de transfert plus faibles que ceux que nous

voL. $13-\mathrm{N}^{\circ} 1$ 
observons sur le maĩs (10 fois moindres). Les raisons de ces différences sont multiples : volume d'eau d'aspersion, port de la plante, forme des feuilles, caractéristiques du tissu des feuilles, comportement du zinc dans la plante. Elles ont pour conséquence un facteur de transfert du ${ }^{65} \mathrm{Zn}$ aux feuilles de maīs plus important. Le maïs fourrage pourrait donc être une voie possible de transfert de ce radionucléide à la ration alimentaire.

\section{TABLEAU IV}

FACTEURS DE TRANSFert dU ${ }^{65} \mathrm{Zn}$ de L'EAU dE RIVIĖre AUX PARTIES AérIENNES DU MAISS IRRIGUÉ PAR ASPERSION

INCIDENCE DE LA TENEUR DE L'EAU EN ZINC ET CADMIUM STABLES (volume d'eau distribuée $300 \mathrm{1} / \mathrm{m}^{2}$ )

\begin{tabular}{|l|c|c|c|}
\hline & \multicolumn{3}{|c|}{$\frac{\mathrm{pCi} / \mathrm{kg} \text { frais }}{\mathrm{pCi} / 1 \text { eau }}$} \\
\cline { 2 - 4 } & Eau de rivière & $\begin{array}{c}\text { Eau de rivière }+\mathrm{Cd} \\
\left(0,010.10^{-6}\right)\end{array}$ & $\begin{array}{c}\text { Eau de rivière }+\mathrm{Zn} \\
\left(5.10^{-6}\right)\end{array}$ \\
\hline Feuilles.......................... & 14,3 & 15,3 & 25,5 \\
Grain....... & 5,3 & 4,3 & 1,4 \\
\hline
\end{tabular}

Aux tableaux III et IV sont présentés deux essaịs sur le maĩs avec du ${ }^{65} \mathrm{Zn}$ sous forme de $\mathrm{Cl}_{2} \mathrm{Zn}$ et en eau de rivière, mais avec des conditions différentes en ce qui concerne le volume d'eau distribué et la protection du sol. On peut constater que les facteurs de transfert du ${ }^{65} \mathrm{Zn}$ de l'eau aux feuilles ne sont pas les mêmes :

- 20,1 avec $400 \mathrm{l} / \mathrm{m}^{2}$ et sans protection du sol;

- 15,3 avec $300 \mathrm{l} / \mathrm{m}^{2}$ et avec protection du sol.

La différence est proportionnelle aux volumes d'eau distribués et par conséquent à la quantité de radionucléide apportée. Celà, nous l'avons déjà observé dans d'autres essais [3]. Il est donc très vraisemblable que l'absorption du ${ }^{65} \mathrm{Zn}$ sous forme de $\mathrm{Cl}_{2} \mathrm{Zn}$ par voie racinaire a été faible. Le transfert par voie foliaire pourrait donc être une voie prépondérante du transfert du ${ }^{65} \mathrm{Zn}$ au maĩs. Or, nous avions observé, dans un précédent essai de contamination du mails par ${ }^{60} \mathrm{Co}$, davantage de mobilité pour la forme complexée que pour la forme chlorure. Cette différence de mobilité s'était traduite par des facteurs de transfert plus élevés de l'eau aux feuilles pour $\mathrm{Cl}_{2} \mathrm{Co}$ que pour Co EDTA [3].

Dans cet essai, l'influence de la forme chimique du radionucléide sur les taux de transfert apparaît comme un phénomène complexe. On constate bien pour le haricot un phénomène identique à celui présenté pour ${ }^{60} \mathrm{Co}$ dans le précédent rapport. Par contre ici, les facteurs de transfert concernant les feuilles de maïs et $\mathrm{Zn}$-EDTA sont nettement plus élevés que ceux qui concernent les mêmes organes et $\mathrm{Cl}_{2} \mathrm{Zn}$. Il se pourrait donc que l'influence de la forme 
chimique d'un radionucléide sur son transfert de l'eau d'irrigation aux plantes soit un problème complexe qui dépende non seulement de la nature de l'élément mais également du végétal considéré.

Les taux de transfert du ${ }^{65} \mathrm{Zn}$ sont plus élevés lorsque la contamination est apportée par l'eau de rivière et ceci pour les deux végétaux : maĩs et haricot. La variation est assez considérable. Dans cet essai, les feuilles sont deux fois plus contaminées par le ${ }^{65} \mathrm{Zn}$ en eau de rivière que lorsque celui-ci est en eau déminéralisée. Les différences au niveau des gousses ou des grains sont moins nettes. Il est vraisemblable que l'eau de rivière contient des produits de nature variée dont les effets sur le ${ }^{65} \mathrm{Zn}$ peuvent être très déterminants. Dans l'expérience suivante où du cadmium et du zinc stables ont été additionnés à l'eau d'irrigation, ceci apparaît nettement (tableau IV).

Le cadmium et le zinc stables présents dans l'eau n'ont pas les mêmes effets sur le transfert du ${ }^{65} \mathrm{Zn}$, et le niveau de ce transfert varie en fonction de la partie du végétal considérée. Le transfert du ${ }^{65} \mathrm{Zn}$ aux feuilles résulte de mécanismes complexes. Les mesures que-nous avons effectuées portaient sur des feuilles non lavées. Cette méthode d'observation masque les phénomènes métaboliques d'incorporation dans les feuilles mais intègre tous les phénomènes de dépôt. Avec ce mode d'observation, il n'apparaît pas que la présence de cadmium influe sur le facteur de transfert du ${ }^{65} \mathrm{Zn}$. Celui-ci reste du même ordre de grandeur que dans le cas où le cadmium n'est pas additionné à l'eau. Le zinc stable, par contre, contribue à élever considérablement le facteur de transfert du ${ }^{65} \mathrm{Zn}$. Tout ce passe comme si le zinc stable jouait le rôle d'entraîneur.

Le facteur de transfert du ${ }^{65} \mathrm{Zn}$ de l'eau aux grains est 2,7 à 3,5 fois moins élevé que ceux qui ont été observés sur les feuilles. Ce taux n'est pas, non plus, influencé significativement par la présence de cadmium aux concentrations tions utilisées $\left({ }^{1}\right)$. La teneur en zinc stable mise en œuvre semble avoir l'effet inverse sur le facteur de transfert aux grains du ${ }^{65} \mathrm{Zn}$ de celui qui a été observé sur les feuilles. Ce transfert est la résultante de deux phénomènes: d'absorption au niveau des feuilles et de migration jusqu'aux grains. Dans ce deuxième phénomène qui fait intervenir des phénomènes biologiques de régulation, l'apport de zinc stable a eu pour effet de diluer le ${ }^{65} \mathrm{Zn}$ qui migre à partir des feuilles.

\section{TRANSFERT DU CADMIUM STABLE}

On constate une fixation du cadmium sur les plantes. Le dépôt sur les feuilles croît quand la teneur en cadmium de l'eau augmente. Cependant, il ne semble pas y avoir proportionnalité entre l'élévation de la teneur en cadmium des feuilles et l'élévation de la teneur en cadmium de l'eau d'arrosage.

( ${ }^{1}$ MYTTENAERE et al. ont montré l'importance du niveau de la concentration de l'eau en cadmium sur le facteur de transport de ${ }^{65} \mathrm{Zn}$ lors d'études de contamination indirecte en écosystème irrigué [7].

voL. $13-\mathrm{N}^{\bullet} 1$ 
Les facteurs de transfert du cadmium aux feuilles semblent donc plus élevés pour les faibles concentrations en cadmium de l'eau. Ce phénomène peut être rapproché de celui qui a été observé chez la tomate sur le plan absorption par voie racinaire [4].

\section{TABLEAU V}

TrANSFERT DIRECT dU CADMIUM STABLe de L'EAU d'ARRosage AU MAIS IRRIGUE PAR ASPERSION

\begin{tabular}{|c|c|c|c|}
\hline & $\begin{array}{c}\text { Teneur } \\
\text { en cadmium } \\
\text { de l'eau } \\
\text { (mg/l) }\end{array}$ & \multicolumn{2}{|c|}{$\begin{array}{c}\text { Teneur en cadmium } \\
\text { des parties aériennes } \\
\text { du maìs } \\
(\mathrm{mg} / \mathrm{kg} \mathrm{sec})\end{array}$} \\
\hline Limites de mesure & 0,004 & \multicolumn{2}{|c|}{0,5} \\
\hline & & Feuilles & Grains \\
\hline 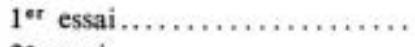 & 0,008 & 1,77 & $<0,5$ \\
\hline $2^{e}$ essai . . . . . . . . . . . . . & 0,044 & 3,27 & $<0,5$ \\
\hline
\end{tabular}

Aucun transfert significatif aux grains n'a été mis en évidence. Le cadmium n'est sans doute pas un élément physiologique indispensable. Bien qu'homologue du zinc, il est peu absorbé par voie foliaire et il est peu mobile dans la plante.

\section{CONCLUSION}

La qualité de l'eau et la forme chimique du radiozinc peuvent intervenir sur le niveau du facteur de transfert de ce polluant au maïs et au haricot, mais différemment selon la partie du végétal considérée.

Les facteurs de transfert du ${ }^{65} \mathrm{Zn}$ aux gousses du haricot sont inférieurs à 1 pour les deux formes chimiques du polluant $\left(\mathrm{Cl}_{2} \mathrm{Zn}-\mathrm{Zn}\right.$ EDTA) et les qualités de l'eau (eau de rivière - eau déminéralisée), La contamination directe par le ${ }^{65} \mathrm{Zn}$, qui peut éventuellement en résulter, n'est pas de nature à augmenter notablement la contamination de la ration alimentaire.

Le ${ }^{65} \mathrm{Zn}$ présente une certaine mobilité dans le maiis, et peut s'accumuler dans les grains de façon plus importante que dans les gousses du haricot, $\mathrm{Ce}$ sont surtout les feuilles qui présentent des taux de transfert élevés (20 à 26 en eau de rivière), L'absorption par voie foliaire semble un facteur prépondérant,

Bien qu'homologues chimiquement, le cadmium et le zinc stables n'exercent pas les mêmes effets sur le transfert du ${ }^{65} \mathrm{Zn}$. Dans nos conditions expérimentales, le cadmium n'a pas d'influence appréciable sur le comportement $\mathrm{du}^{65} \mathrm{Zn}$. Par contre, les répercussions dues à la présence du zinc stable dans l'eau d'irrigation sur le taux de transfert du ${ }^{65} \mathrm{Zn}$ au maĩs sont importantes. 
Elles sont aussi de sens contraire si l'on considère les feuilles d'une part et les graines d'autre part. Le zinc stable fait croitre le taux de transfert aux feuilles. Il agit alors comme entraîneur dans les mécanismes de fixation sur les tissus superficiels de ces organes. Il fait diminuer le facteur de transfert aux grains en diluant le ${ }^{65} \mathrm{Zn}$ qui migre à partir des feuilles.

Le cadmium de l'eau d'irrigation appliquée au maïs se fixe sur les feuilles. Par contre, on ne constate pas de migration significative du cadmium des feuilles aux grains pour les concentrations mises en œuvre dans l'expérimentation.

Le rôle important que joue le maīs fourrage dans l'économie agricole, ses besoins en eau, la capacité de fixation des feuilles vis-à-vis du zinc et du cadmium, font du maĩs un vecteur possible du transfert de ces produits à la ration alimentaire des animaux d'élevage.

\section{BIBLIOGRAPHIE}

[1] Perkins R. W., Niflsen J. M., Zinc 65 in foods and people. Science, 1959, 129, 94-95.

[2] Perkins R. W., Nielsen J. M., Roesch W. C., McCall R. C., Zine 65 and chromium 51 in foods and people. Science, 1960, 132, 1895-1897.

[3] Delmas J., Grauby A., Disdier R. Études expérimentales sur le transfert dans les cultures de quelques radionucléides présents dans les effluents des centrales électronucléaires. In : Environmental behaviour of radionuclides released in the nuclear industry, Aix-en-Provence, 14-18 May 1973. Vienne, A.I.E.A., 1973, 321-332.

[4] Sterner A. A., Uptake of cadmium by plants. Landbouwkd. Tijdschr., 1973, 85, 4, 124-128.

[5] Cox C. R. Techniques et contrôle du traitement des eaux. O.M.S., Genève, 1967, 191-192.

[6] United States, Department of Interior, Federal Water Pollution Control Administration. Report of the Committee on water quality criteria, Washington, 1968.

[7] Myttennaere C., Merlini M., Bittel R., Dabin P., Mousny J. M., Pozzi G. Étude de l'influence du cadmium stable sur le transfert du zine-65 en écosystème irrigué par submersion (rizière irriguée). In : Impact of nuclear releases into the aquatic environment, Otaniemi, 30 June-4 July 1975. Vienne, A.I.E.A., 1975, 39-47. 